J. Indones. Math. Soc.

Special Edition (2011), pp. 133-136.

\title{
HOW PROVABLY GRACEFUL ARE THE TREES?
}

\author{
Peter J. Slater
}

${ }^{1}$ Mathematical Sciences Dept. and Computer Science Dept., University of Alabama Huntsville, AL, USA 35899 slaterp@uah.edu and pslater@cs.uah.edu

\begin{abstract}
Given the inability to prove that all trees are graceful, can we at least say something positive about their gracefulness? Two problems of this type are presented.

Key words: Bijection, tree, graceful.
\end{abstract}

2000 Mathematics Subject Classification: 05C78

Received: 09-08-2011, revised: 09-09-2011, accepted: 04-12-2012. 